\title{
CHARACTERISTICS OF ELECTRON ACCELERATION IN A SELF-MODULATED LASER WAKEFIELD*
}

\author{
S.-Y. Chen, M. Krishnan, A. Maksimchuk and D. Umstadter ${ }^{\dagger}$
}

\begin{abstract}
The electron beam generated in a self-modulated laser wakefield accelerator is characterized. It was observed to have a multi-component beam profile and its energy distribution undergoes discrete transitions as the laser power or plasma density is varied. In addition, dark spots that form regular modes were observed in the electron beam profile.
\end{abstract}

\section{INTRODUCTION}

Acceleration of electrons by an electron plasma wave has been of great current interest because of its much larger acceleration gradient than that of conventional rf linacs [1]. Several methods have been proposed for driving a largeamplitude fast-phase-velocity plasma wave [1]. In the Laser Wake-Field Accelerator (LWFA), an electron plasma wave is driven resonantly by a short laser pulse through longitudinal laser ponderomotive force [2]. In the SelfModulated Laser Wake-Field Accelerator (SMLWFA), an electron plasma wave is excited by a relatively long laser pulse undergoing stimulated Raman forward scattering instability $[3,4,5]$. The injection of electrons can occur by trapping of hot background electrons, which are preheated by other processes such as Raman backscattering and sidescattering instabilities $[6,7,8]$, or by wavebreaking (longitudinal [1] or transverse [9]). It can also be achieved by specific injection schemes $[10,11]$ in order to control the characteristics of the generated electron beam.

Several groups have observed the generation of $\mathrm{MeV}$ electrons from the SMLWFA [7, 8, 12, 13, 14, 15]. In this experiment, the electron beam produced from a selfmodulated laser wakefield accelerator injected with selftrapping of electrons was characterized in detail. The observations of up-to-three-component electron-beam profiles and up-to-two discrete changes in the slope of electron energy distribution are reported. In addition, dark spots that form regular modes were observed in the first beam-profile component. These new observations provide us important new clues to the underlying dynamics of electron acceleration in a three-dimensional (3-D) plasma wave.

\section{EXPERIMENTAL SETUP}

The experiment was done by using a Ti:sapphire/Nd:glass laser system that produced 400 -fs-duration laser pulses at $1.053-\mu \mathrm{m}$ wavelength with a maximum peak power of 4

\footnotetext{
${ }^{*}$ Supported by the Division of High Energy Physics, Office of Energy Research, U.S. Department of Energy, award DE-FG02-98ER41071, with facilities supported by the National Science Foundation.

$\dagger$ Center for Ultrafast Optical Science, University of Michigan, Ann Arbor, MI 48109
}

TW. The 50-mm-diameter laser beam was focused with an f/3.3 parabolic mirror onto the front edge of a supersonic helium gas jet. The focal spot in vacuum was a 7- $\mu \mathrm{m}-$ FWHM near-Gaussian spot, which contained $60 \%$ of the total pulse energy, and a large dim spot $(100 \mu \mathrm{m}$ FWHM). The helium gas was fully ionized by the foot of the laser pulse. At a laser power of $\geq 2 \mathrm{TW}$ and a plasma density of $\geq 2 \times 10^{19} \mathrm{~cm}^{-3}$, the laser pulse underwent relativisticponderomotive self-channeling $[13,16]$, and the laser channel of $<10-\mu \mathrm{m}$ diameter extended to be $750 \mu \mathrm{m}$ in length, the length of the gas jet. An electron plasma wave was excited by the laser pulse through stimulated Raman forward scattering instability, as was evident from the observation of Raman satellites in the transmitted light [7]. An electron beam was produced, when the laser power or gas density exceeded a certain threshold, and propagated in the direction of the laser beam. The spatially-averaged time-resolved plasma-wave amplitude was measured using collinear collective Thomson scattering [17]. The peak plasma-wave amplitude was observed to increase with increase of laser power or plasma density, and the maximum observed was about $30 \%$ density perturbation.

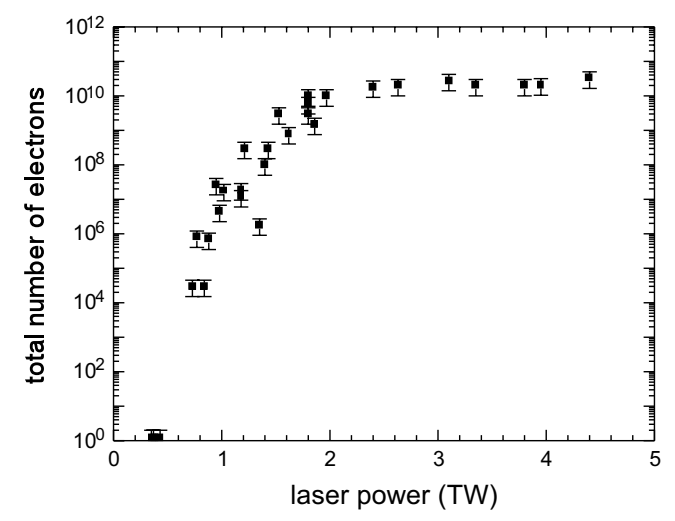

Figure 1: Total number of electrons with energies $>60 \mathrm{keV}$ in the generated electron beam as a function of laser power at $3.7 \times 10^{19}-\mathrm{cm}^{-3}$ plasma density.

The electron energy spectrum in the low energy range $(<8 \mathrm{MeV})$ was measured using a dipole permanent magnet with a LANEX scintillating screen imaged by a CCD camera as the detector. Higher-energy electron energy spectra were obtained by using dipole electro-magnets and a multi-wire proportional chamber (MWPC). The electronbeam spatial profile at $16 \mathrm{~cm}$ away from the gas jet was measured using a LANEX scintillating screen imaged by a CCD camera. Because of the aluminum foil in front of the LANEX, which was used for blocking the laser light, only 
electrons with kinetic energies higher than $100 \mathrm{keV}$ were imaged. Since the source size of the generated electron beam was small, $\sim 10 \mu \mathrm{m}$ in diameter, the electron beam profile on the LANEX was actually a measurement of the angular pattern. The total number of electrons in the beam was measured either by using a Faraday cup to directly collect the entire electron beam or by using a collimator and a calibrated scintillator-PMT (photomultiplier tube).

\section{RESULT AND DISCUSSION}

\section{$3.1 \quad$ Number of Electrons}

Figure 1 shows the total number of electrons in the beam as a function of laser power at a fixed plasma density [7]. Below a threshold, no electron is observed. When the laser power exceeds this threshold, the number of electrons increases exponentially with increase of laser power, and then gradually saturates. The variation of the number of electrons with change of plasma density shows the same behavior. The threshold of the electron production is believed to be associated with the electron trapping threshold.

\subsection{Transverse Beam Profile}
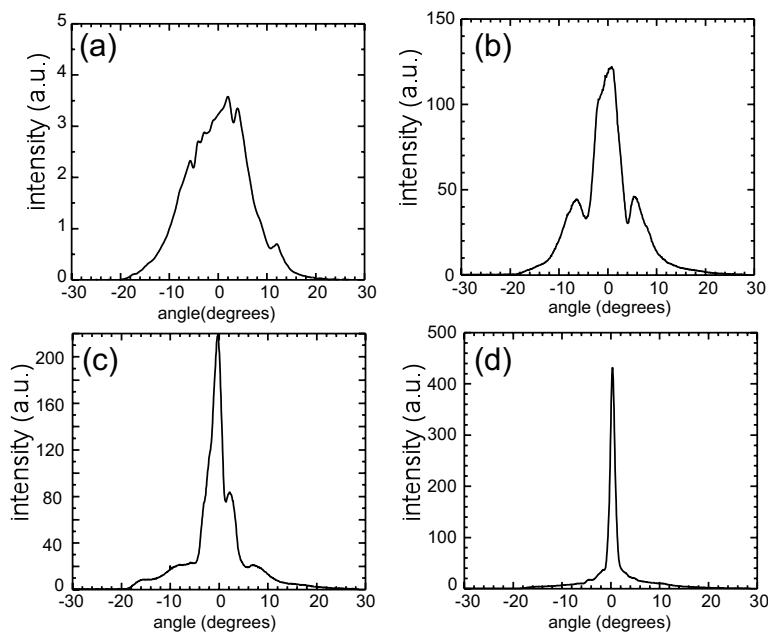

Figure 2: Lineouts of the electron beam profiles for various laser powers at $2.3 \times 10^{19}-\mathrm{cm}^{-3}$ plasma density: (a) 0.6 TW, (b) $1.1 \mathrm{TW}$, (c) $2.0 \mathrm{TW}$, and (d) $2.9 \mathrm{TW}$.

The electron beam profile (angular pattern) was observed to contain several concentric Gaussian-like-profile beams, and the number of beam components depends on laser power and plasma density. For a plasma density of $2.3-6.2 \times 10^{19} \mathrm{~cm}^{-3}$, only one beam component $\left(\sim 20^{\circ}\right.$ FWHM) exists in the electron beam at 0.6-TW laser power. At a laser power larger than $1 \mathrm{TW}$, a second beam component grows up on top of the first beam component, with a divergence angle of $5^{\circ}-10^{\circ}$ FWHM. For $2.3-3.4 \times 10^{19}$ $\mathrm{cm}^{-3}$ plasma density, a third beam component with a divergence angle of $1^{\circ}-3^{\circ}$ FWHM appears when the laser power is higher than 2 TW. Figure 2 shows the lineouts of the electron beam profiles for various laser powers at $2.3 \times 10^{19}-\mathrm{cm}^{-3}$ plasma density. Basically, the divergence angle of the second beam component increases and that of the third beam component decreases with increase of laser power, while the divergence angle of the first beam component is roughly invariant $\left(20^{\circ}-25^{\circ}\right)$. The multiplecomponent electron beam profile is believed to result from the transverse electric field of the plasma wave.
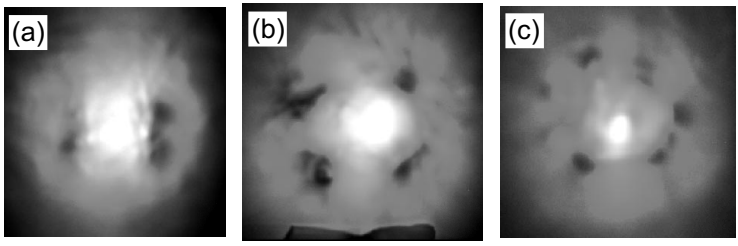

Figure 3: Images of the transverse electron beam profiles at various laser powers and plasma densities: (a) $1.1 \mathrm{TW}$, $3.4 \times 10^{19} \mathrm{~cm}^{-3}$, (b) $3.5 \mathrm{TW}, 6.2 \times 10^{19} \mathrm{~cm}^{-3}$, and (c) 2.0 TW, $2.3 \times 10^{19} \mathrm{~cm}^{-3}$.

The appearance of the second beam component was observed to coincide with the sudden extension of the plasmawave channel (which is determined by the laser channel) caused by laser self-guiding. In addition, when the second beam component appears, there are usually some holes appearing in the first beam component, as shown in Fig. 3. These holes form regular patterns that are similar to $\mathrm{TM}_{12}, \mathrm{TM}_{22}$ and $\mathrm{TM}_{32}$ electromagnetic modes in a circular waveguide, or to $(1,0),(1,1)$ and $(1,2)$ HermiteGaussian modes of a laser beam. Furthermore, under the highest laser power and plasma density in this experiment, a density depression at the center of the accelerated electron beam was observed occasionally, as shown in Fig. 4. The dark modes appearing in the electron beam profile may be caused by the excitation of electron plasma waves with higher-order modes or with density depressions.

\subsection{Electron Energy Spectrum}
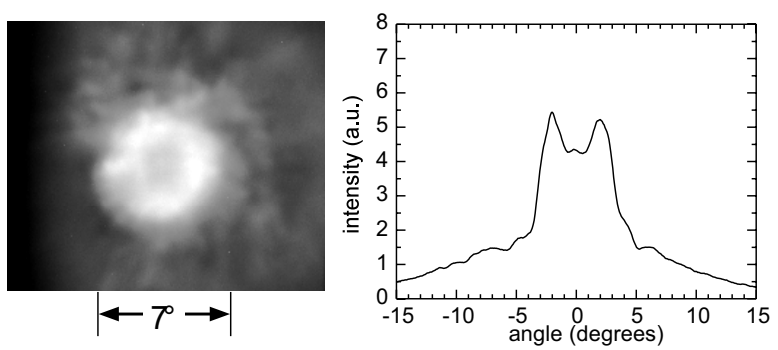

Figure 4: Image and vertical lineout of the transverse electron beam profile at 3.5-TW laser power and $6.2 \times 10^{19}$ $\mathrm{cm}^{-3}$ plasma density.

Figure 5 shows the normalized electron energy spectra in 
the low energy range for various laser powers and plasma densities. The spectra were found to have Maxwellian-like distributions, i.e., $\exp (-\alpha \gamma)$, where $\gamma$ is the relativistic factor of electron energy. The slope, $\alpha$, of the spectrum was found to change discretely with variation of laser power and plasma density. For instance, at a fixed plasma density, the slope remains the same with increasing laser power until a certain threshold is reached. Then the slope $\alpha$ changes to a lower value, and stays the same with further increase of laser power until the next jump. The same behavior occurs for varying plasma density at a fixed laser power. Three $\alpha$ values were observed in this experiment: 1.0, 0.6 and 0.3 . The occurrence of the first jump coincides with the extension of the laser channel from $<400 \mu \mathrm{m}$ to $750 \mu \mathrm{m}$; however, when the second jump occurs, there is no change in the laser channel length. The slopes of the electron energy spectra in the low energy range were found to be the same for these three beam components. The transition of the slope may be related to the ratio between the plasmawave channel length and the electron detuning length.

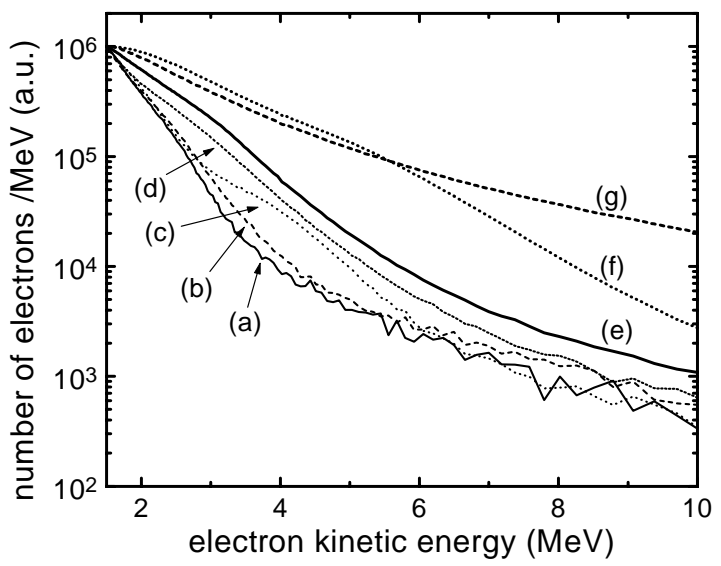

Figure 5: Electron energy spectra for various laser powers and plasma densities: (a) $2.6 \mathrm{TW}, 3.4 \times 10^{19} \mathrm{~cm}^{-3}$, (b) 2.9 $\mathrm{TW}, 3.5 \times 10^{19} \mathrm{~cm}^{-3}$, (c) $3.3 \mathrm{TW}, 4.8 \times 10^{19} \mathrm{~cm}^{-3}$, (d) $3.9 \mathrm{TW}, 4.8 \times 10^{19} \mathrm{~cm}^{-3}$, (e) $1.7 \mathrm{TW}, 6.2 \times 10^{19} \mathrm{~cm}^{-3}$, (f) $2.7 \mathrm{TW}, 6.2 \times 10^{19} \mathrm{~cm}^{-3}$, and (g) $3.5 \mathrm{TW}, 6.2 \times 10^{19}$ $\mathrm{cm}^{-3}$.

Another important observation in this experiment is a two-temperature distribution in the electron energy spectrum. As shown in Fig. 6, which was obtained using both the low-energy and high-energy electron spectrometers, the slope of electron energy distribution in the low energy range $(\leq 5 \mathrm{MeV})$ is steep, while the slope in the high energy range is much less steep (almost flat). The two-slope electron energy spectrum is believed to be a result of electron motion inside and outside the separatrix.

\subsection{Other Parameters}

The temporal duration of the electron pulse should be roughly equal to that of the plasma wave, $\sim 2$ ps [17], since the former is generated from the latter. The separation be-

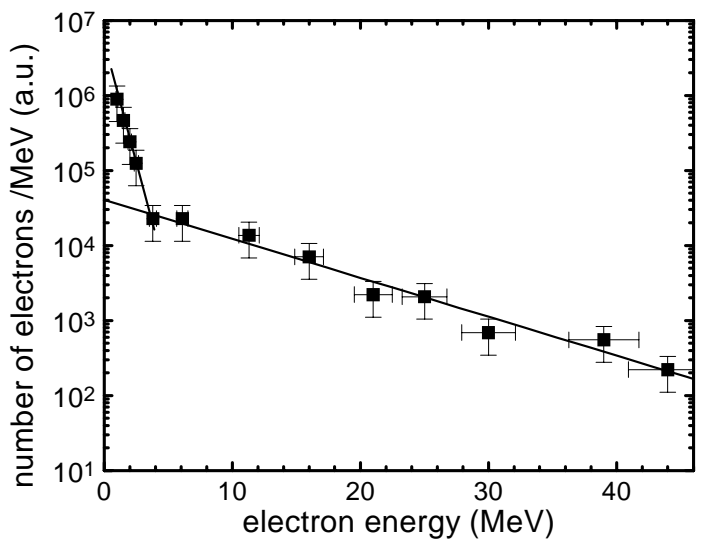

Figure 6: Electron energy spectrum for 3-TW laser power and $3 \times 10^{19}-\mathrm{cm}^{-3}$ plasma density. The solid lines represent exponential fits.

tween adjacent micro bunches is equal to the plasma-wave period, which is $18 \mathrm{fs}$ for a plasma density of $3.4 \times 10^{19}$ $\mathrm{cm}^{-3}$. The duration of a micro bunch should be less than that. Therefore, the maximum peak current is estimated to be about $1 \mathrm{kA}$ or higher. The transverse cross section of the electron source should be roughly equal to the cross section of the plasma wave, which roughly equals that of the laser beam, $\sim 10 \mu \mathrm{m}$. In the best cases, the angular divergence of the electron beam was measured to be $\sim 1^{\circ}$, which leads to a normalized transverse emittance of 0.06 $\pi$-mm-mrad for $2 \mathrm{MeV}$ electrons.

\section{REFERENCES}

[1] See references in E. Esarey et al., IEEE Trans. Plasma Sci. PS-24, 252 (1996).

[2] T. Tajima and J. M. Dawson, Phys. Rev. Lett. 43, 267 (1979).

[3] P. Sprangle et al., Phys. Rev. Lett. 69, 2200 (1992).

[4] T. M. Antonsen, Jr. and P. Mora, Phys. Rev. Lett. 69, 2204 (1992).

[5] N. E. Andreev et al., JETP Lett. 55, 571 (1992).

[6] P. Bertrand et al., Phys. Rev. E 49, 5656 (1994).

[7] D. Umstadter et al., Science 273, 472 (1996).

[8] C. I. Moore et al., Phys. Rev. Lett. 79, 3909 (1997).

[9] S. V. Bulanov et al., Phys. Rev. Lett. 78, 4205 (1997).

[10] D. Umstadter, J. K. Kim, and E. Dodd, Phys. Rev. Lett. 76, 2073 (1996).

[11] E. Esarey et al., Phys. Rev. Lett. 79, 2682 (1997).

[12] A. Modena et al., Nature 377, 606 (1995).

[13] R. Wagner et al., Phys. Rev. Lett. 78, 3125 (1997).

[14] G. Malka et al., Phys. Rev. Lett. 79, 2053 (1997).

[15] D. Gordon et al., Phys. Rev. Lett. 80, 2133 (1998).

[16] S.-Y. Chen et al., Phys. Rev. Lett. 80, 2610 (1998).

[17] S. P. Le Blanc et al., Phys. Rev. Lett. 77, 5381 (1996). 